\title{
Helping cells to remember
}

\begin{abstract}
Differentiated cells 'remember' their identity even when they find themselves in a new cellular environment. This ability to maintain the correct genetic programme after cell division has previously been attributed to various epigenetic factors, which in most cases have been shown to remember a repressed state. New work from $\mathrm{Ng}$ and Gurdon now shows that histone $\underline{\mathrm{H} 3.3}$ is required for the persistence of some active gene states.

Building on their previous work, $\mathrm{Ng}$ and Gurdon carried out serial nuclear transfer in Xenopus laevis using endoderm nuclei as the donors. Their results showed that epigenetic memory is remarkably stable
\end{abstract}

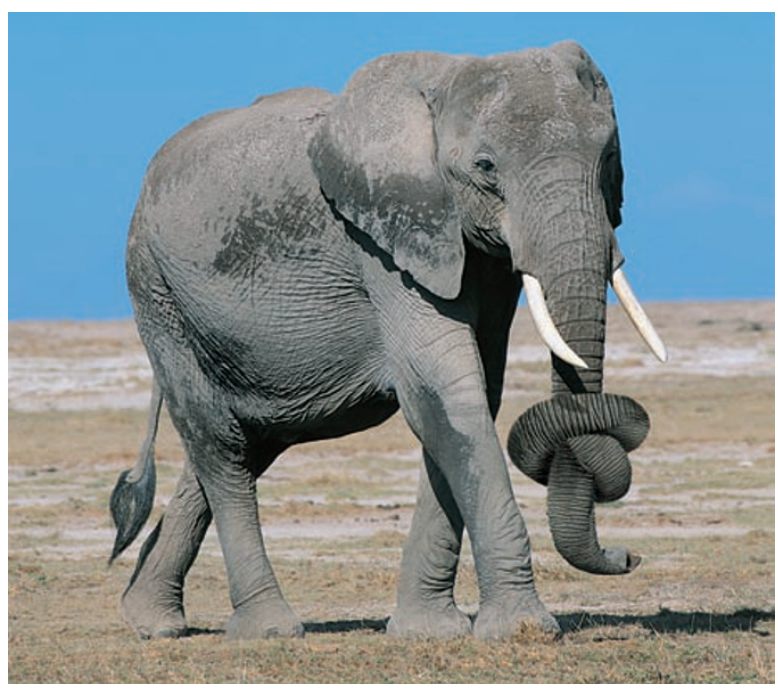

DIGITAL VISION
- expression of the endodermal gene edd in the neuroectodermal lineage persisted after two rounds of nuclear transfer and 24 mitoses, and in the absence of transcription.

Seeking the basis of this memory, the authors discounted DNA methylation - reducing genome-wide methylation levels by up to $60 \%$ had no effect on expression either from the edd locus or on $M y o D$, which the authors selected because of its wellcharacterized promoter.

Next, Ng and Gurdon turned their attention to histone H3.3, which is enriched in actively expressed genes. Indeed, H3.3 was abundant in the MyoD promoter in cells that express this gene but not in other cells. Moreover, chromatin immunoprecipitation analysis revealed that embryos in which the epigenetic memory of $M y o D$ expression persists following nuclear transfer have a higher level of H3.3 in the $M y o D$ promoter than embryos in which this memory has been lost.

The authors turned this correlation between $\mathrm{H} 3.3$ levels and memory of expression into causality in a series of experiments. They incorporated into the $M y o D$ promoter a mutant form of $\mathrm{H} 3.3$ in which lysine 4 was replaced with glutamic acid - abolishing a key H3.3 methylation site - and found that, on nuclear transfer, memory of
MyoD expression was lost. Moreover, whereas overexpressing wild-type H3.3 in nuclear transfer embryos enhanced memory, overexpression of the mutant form led to memory reduction. Therefore, the authors propose that the incorporation of H3.3 methylated at lysine 4 into a particular locus - for example, in response to differentiation factors is the basis for the cellular memory of active expression states.

Having also assessed epigenetic memory at other genes that are expressed at various stages in the muscle lineage, the authors reason that this type of memory occurs in cells that are committing to a developmental pathway, but not at loci involved in terminal differentiation. The authors conclude that "the stable inheritance of cellular memory might provide an explanation for the decreasing success of nuclear transfer from more differentiated donor cell nuclei”.

\section{Magdalena Skipper}

ORIGINAL RESEARCH PAPER Ng, R. K. \& Gurdon, J. B. Epigenetic memory of an active gene state depends on histone $\mathrm{H} 3.3$

incorporation into chromatin in the absence of transcription. Nature Cell Biol. 9 December 2007 (doi: 10.1038/ncb1674)

FURTHER READING Henikoff, S. Nucleosome destabilization in the epigenetic regulation of gene expression. Nature Rev. Genet. 4 December 2007 (doi:10.1038/nrg2206)|Schwartz, Y. B. \& Pirrotta, V. Polycomb silencing mechanisms and the management of genomic programmes. Nature Rev. Genet. 8, 9-22 (2007) 